\title{
INFLUÊNCIA DE EXERCÍCIOS POSTURAIS SOBRE A FORÇA MUSCULAR RESPIRATÓRIA EM JOVENS SAUDÁVEIS
}

Aline Barbosa Silva, Gabriela Araújo Caldeira, Jaíne Lopes Ferreira, Rodrigo Martins Dias, Maria Tereza Artero Prado Dantas, Deborah Cristina Gonçalves Luiz Fernani

Universidade do Oeste Paulista - UNOESTE, Presidente Prudente, SP. e-mail: gabriela.gac54@gmail.com

\section{RESUMO}

Alterações posturais representam um problema de saúde pública que pode interferir de forma direta na mecânica ventilatória. Exercícios de Pilates e isostretching podem ser benéficos para a melhora postural e ventilatória. Os objetivos deste estudo foram analisar efeitos de exercícios combinados dos métodos de isostretching e Pilates sobre a força muscular respiratória (FMR), função pulmonar e alterações posturais. A postura, FMR e função pulmonar de 20 indivíduos foram avaliadas e reavaliadas após um treinamento de 10 semanas. Os 20 indivíduos foram classificados de acordo com índice de massa corporal (IMC) e nível de atividade física. Quando comparados os valores pré e pós-treinamento observou-se melhora significante da FMR. A PiMáx apresentou aumento significante $(p=0,002)$ da mesma forma que a PeMáx $(p=0,006)$. Porém, não foram observadas mudanças estatisticamente significantes na postura destes participantes. Conclui-se que há melhora da FMR, realizando o treinamento com exercícios posturais uma vez por semana associados a exercícios para mecânica ventilatória, da mesma forma que PFE e CVF também tiveram resultados positivos, porém em relação à postura não houve melhora considerável nos resultados.

Palavras-chave: postura, exercício, músculos respiratórios, espirometria.

\section{INFLUENCE OF POSTURAL EXERCISES ON RESPIRATORY MUSCLE FORCE IN HEALTHY YOUNG PEOPLE}

\begin{abstract}
Postural changes represent a public health problem that can interfere directly with ventilatory mechanics. Pilates and isostretching exercises may be beneficial for postural and ventilatory improvement. The objectives of this study were to analyze effects of combined exercises of the isostretching and Pilates methods on respiratory muscle strength (FMR), lung function and postural changes. The posture, FMR and lung function of 20 individuals were evaluated and reevaluated after a 10-week training. The 20 subjects were classified according to body mass index (BMI) and level of physical activity. When the pre and post training values were compared, improvement of the significant FMR was observed. The maxIP showed a significant increase $(p=0.002)$ in the same way as the maxEP $(p=0.006)$. However, no statistically significant changes were observed in the posture of these participants. It was concluded that there is an improvement of the FMR, performing the training with postural exercises once a week associated with exercises for ventilatory mechanics, just as PEF and FVC also had positive results, but in relation to the posture there was no significant improvement in the results.
\end{abstract}

Keywords: posture, exercise, respiratory muscles, spirometry. 


\section{INTRODUÇÃO}

Força muscular (FM) é descrita como a tensão gerada em um músculo decorrente de uma contração, sendo um fator de extrema importância na realização das atividades de vida diária, promovendo independência, mantendo a aptidão física e a saúde do individuo ${ }^{1}$. No entanto, a redução da FM pode tornar o ser humano incapaz de realizar algumas atividades como, andar, correr, realizar suas atividades laborais, entre outras situações, fato que pode desencadear um ciclo vicioso, no qual, pelo desuso da musculatura ocorrerá hipotrofia da mesma, redução de força e incapacidade ${ }^{2}$.

Neste sentido, a FM também é a responsável pela sustentação de estruturas corporais, como a coluna vertebral, acarretando estabilidade para a mesma durante os movimentos realizados, $O$ que pode ser denominado de postura ${ }^{3}$. A diminuição da FM pode afetar a postura do ser humano, fato que pode gerar dor e desgaste das estruturas do sistema músculo esquelético ${ }^{4,5}$, pelas ações compensatórias que o corpo adquire para permanecer em equilíbrio ${ }^{6}$.

Os problemas decorrentes de alterações posturais são atualmente questões de saúde publica $^{7,8}$, pois apresentam grande incidência, tornando este um fator preocupante, devido ser causador de incapacidades a curto e longo prazo, sendo necessário o desenvolvimento de politicas públicas voltadas a reduzir os maus hábitos posturais ${ }^{3}$, principalmente durante a infância e juventude ${ }^{3,8,9}$.

Dentre os problemas causados pelas alterações posturais, podemos encontrar aqueles que comprometem a mecânica ventilatória, em virtude das alterações na conformação da caixa torácica, que podem acarretar alterações na função pulmonar ocasionando um distúrbio ventilatório restritivo com redução da capacidade vital funcional $(\mathrm{CVF})^{13}$. Além disso, podemos destacar alterações no posicionamento dos músculos respiratórios, o que pode torná-los encurtados ou alongados, ocasionando prejuízos à mecânica respiratória com perda de força muscular respiratória (FMR) como consequência ${ }^{13}$.

A perda da FMR pode ser identificada por meio da manovacuometria, que se trata de um método simples para a avaliação da força dos músculos respiratórios ${ }^{14}$, enquanto que a função pulmonar pode ser avaliada por meio da espirometria, na qual é possível verificar os volumes e capacidades pulmonares, o que auxilia na quantificação dos distúrbios ventilatórios ${ }^{15}$.

Os exercícios baseados nos métodos de isostretching $^{16,17}$ e Pilates ${ }^{18,19}$, por sua vez, apresentam influência comprovada sobre a força muscular respiratória ${ }^{13,18}$ e são muito utilizados na prática clínica. Porém, esses métodos são geralmente utilizados de forma não combinada, para o tratamento de problemas relacionados a postura. Dessa forma, escassos são os estudos relacionando esses exercícios de forma combinada, à mecânica ventilatória.

O pilates é uma técnica baseada nos princípios de técnicas de exercício como ioga, artes marciais e meditação, cujo intuito é o controle dos músculos envolvidos nos movimentos da forma mais consciente possível com o uso da concentração, controle, centragem, respiração diafragmática, leveza, precisão, força e relaxamento ${ }^{20}$. Já o método de Isostretching tem o intuito de trabalhar com manutenção de posturas ao realizar $o$ ato expiratório. Esse método usa exercícios de alongamento e contrações isométricas ${ }^{21}$.

Neste contexto, há a necessidade de novos estudos que procurem analisar os efeitos da alteração da postura sobre a FMR e função pulmonar, e, os possíveis efeitos de exercícios terapêuticos aplicados para o tratamento postural.

O objetivo deste estudo foi analisar os efeitos de um programa de exercícios combinados dos métodos de isostretching e Pilates sobre a FMR, função pulmonar e alterações posturais em indivíduos na transição da adolescência para a vida adulta.

\section{METODOLOGIA}

Foi realizado um estudo longitudinal, aprovado pelo comitê de ética em pesquisa (CAAE: 58175116.6.0000.5515), no qual os indivíduos da amostra assinaram termo de consentimento livre e esclarecido.

A amostra foi composta inicialmente por 20 indivíduos participantes de um projeto de extensão para capacitação do tratamento postural, com média de idade de $18,2 \pm 0,4$ anos, sendo sete do sexo masculino e 13 do feminino. Os indivíduos da amostra foram caracterizados de acordo com o Sistema de Vigilância Alimentar e Nutricional (SISVAN) ${ }^{22}$ e o nível de atividade física dos indivíduos também foi analisado por meio da aplicação do Questionário Internacional 
de Atividade Física, IPAQ - International Physical Activity Questionarie ${ }^{23}$.

Foram estabelecidos como critérios de inclusão a idade, entre 18 e 19 anos e não possuir doenças respiratórias crônicas. Já para os critérios de exclusão estabeleceu-se os que não completaram todas as etapas do projeto.

Para análise do perfil postural foi utilizado o Instrumento de Avaliação Postural (IAP) de Liposcki et al. ${ }^{24}$, o qual é um formulário de análise postural, que verifica desalinhamentos dos segmentos corporais como tronco e membros, em diferentes ângulos de observação como vista anterior, lateral e posterior, sendo preenchido de acordo com a análise visual direta do avaliador, posicionados a dois metros do indivíduo.

Para avaliação da FMR foi utilizado um Manovacuômetro analógico (Comercial Medical ${ }^{\circ}$ ), com escalas de $-120 \mathrm{a}+120 \mathrm{cmH} 2 \mathrm{O}$, e por este foram obtidas a pressão inspiratória máxima (PiMáx) e pressão expiratória máxima $(\text { PeMáx })^{25,26}$. As medidas foram realizadas no máximo cinco vezes e com sustentação máxima de 3 segundos ${ }^{14,25,26}$, sendo adotada a maior desde que não houvesse variação de $10 \%$ entre a maior e menor.

Para avaliar a função pulmonar foi realizada a espirometria, por meio de um espirômetro (MicroLoop Care Fusion), acoplado a um computador. Os critérios de aceitação e reprodutibilidade estão de acordo com as normas da American Thoracic Society e European Respiratory Society ${ }^{27}$ e com valores de normalidade relativos à população brasileira ${ }^{15}$.

Os jovens foram submetidos a um protocolo de exercícios posturais com associação da filosofia de tratamento dos métodos de Isostretching ${ }^{28,29}$ e Pilates ${ }^{2,30}$ desenvolvidos em 10 sessões de uma hora com frequência de uma vez na semana, constituídas por exercícios de flexibilidade, fortalecimento muscular, manutenção postural e exercícios respiratórios, com uso de bola, bastão e faixa elástica. Dentre eles pontua-se exercícios de ponte ${ }^{26}$, diafragmático com contração isométrica do assoalho pélvico ${ }^{31}$ auto crescimento ${ }^{16,31}$, abdominais ${ }^{18,31}$, além de exercícios isométricos e isotônicos de membros superiores e inferiores ${ }^{32}$.

Os exercícios foram realizados na posição deitada, e em maioria sentada e em pé, por 10 repetições cada, sendo o tempo de cada repetição determinado pela expiração prolongada do indivíduo, com intervalo de repouso de 30 segundos entre eles.

As reavaliações ocorreram nos mesmos moldes da avaliação: perfil postural, testes de FMR, função pulmonar, com exceção dos indicadores antropométricos e do nível de atividade física.

Cada elemento da avaliação e da reavaliação deste estudo foi desenvolvido por um único avaliador previamente treinado, sendo que o mesmo ocorreu com o treinamento a fim de padronizar os achados.

Para análise dos dados foi utilizado o software estatístico SPSS versão 15.0. Os dados quantitativos foram expressos em média e desvio padrão para dados normais e mediana e intervalo interquartílico (25-75\%) para dados não normais (normalidade testada pelo teste de Shapiro-Wilk). Já dados qualitativos foram expressos em frequência e percentual. Para análise intragrupos dos dados quantitativos (antes e após o treinamento) foi utilizado teste $t$ de Student para amostras pareadas ou teste de Wilcoxon, dependendo da normalidade dos dados. O teste McNemar foi utilizado para analise dos dados qualitativos com relação a avaliação postural. Em seguida para avaliar a relação entre os valores espirométricos e força muscular respiratória no momento pós treinamento foi aplicado o coeficiente de correlação de Spearman. Foram considerados significativos os valores de $p<0,05$.

\section{RESULTADOS}

A amostra foi composta por 20 indivíduos, dos quais 15 foram classificados em eutrofia o que corresponde à $75 \%$ do total de participantes, 4 indivíduos estavam em sobrepeso correspondendo à $20 \%$ e 1 em obesidade que resulta em $5 \%$ do número total ${ }^{22}$. Além de apresentarem como média de circunferência abdominal $76,4 \pm 11,8 \mathrm{~cm}$. Do número, 2 participantes estavam com o nível de atividade física insuficientemente ativos, 10 eram ativos e 8 indivíduos muito ativos ${ }^{23}$.

Quanto à postura dos jovens, não houve significância estatística para nenhuma das variáveis avaliadas quando comparadas nos momentos antes e depois do programa de exercícios, porém ainda assim apresenta resultados com importância clínica para os profissionais da área (Tabela 1). 
Tabela 1. Efeitos do programa de exercícios sobre as alterações posturais

\begin{tabular}{|c|c|c|c|}
\hline$N=20$ & Antes & Depois & $p$ \\
\hline \multicolumn{4}{|l|}{ Vista anterior cabeça } \\
\hline Normal & 20 & 20 & NA \\
\hline \multicolumn{4}{|l|}{ Vista lateral cabeça } \\
\hline Normal & 18 & 17 & \multirow{2}{*}{1,000} \\
\hline Para frente & 2 & 3 & \\
\hline \multicolumn{4}{|l|}{$\begin{array}{l}\text { Vista anterior do } \\
\text { ombro }\end{array}$} \\
\hline Simétrico & 13 & 12 & \multirow{3}{*}{0,368} \\
\hline Elevado para direita & 4 & 6 & \\
\hline Elevado para esquerda & 3 & 2 & \\
\hline \multicolumn{4}{|l|}{ Vista lateral do ombro } \\
\hline Simétrico & 14 & 17 & \multirow{2}{*}{0,375} \\
\hline Elevado para direita & 6 & 3 & \\
\hline \multicolumn{4}{|l|}{ Triangulo de Thales } \\
\hline Simétrico & 15 & 15 & \multirow{3}{*}{0,513} \\
\hline $\begin{array}{l}\text { Assimétrico para } \\
\text { direita }\end{array}$ & 4 & 3 & \\
\hline $\begin{array}{l}\text { Assimétrico para } \\
\text { esquerda }\end{array}$ & 1 & 2 & \\
\hline \multicolumn{4}{|l|}{ Tronco } \\
\hline Alinhado & 19 & 18 & \multirow{3}{*}{ NA } \\
\hline Rotação a direita & 0 & 2 & \\
\hline Rotação a esquerda & 1 & 0 & \\
\hline \multicolumn{4}{|l|}{ Coluna cervical } \\
\hline Normal & 18 & 18 & \multirow{3}{*}{ NA } \\
\hline Hiperlordose & 2 & 1 & \\
\hline Retificação & 0 & 1 & \\
\hline \multicolumn{4}{|l|}{ Coluna torácica } \\
\hline Normal & 18 & 18 & \multirow{3}{*}{1,000} \\
\hline Hipercifose & 1 & 1 & \\
\hline Retificação & 1 & 1 & \\
\hline \multicolumn{4}{|l|}{ Coluna lombar } \\
\hline Normal & 18 & 16 & \multirow{3}{*}{0,157} \\
\hline Hiperlordose & 1 & 3 & \\
\hline Retificação & 1 & 1 & \\
\hline \multicolumn{4}{|l|}{ Coluna vertebral } \\
\hline Escoliose "S" & 6 & 6 & \multirow{3}{*}{0,228} \\
\hline Escoliose "C" & 4 & 2 & \\
\hline Normal & 10 & 12 & \\
\hline \multicolumn{4}{|l|}{ Escapula } \\
\hline Alada a direita & 2 & 1 & \multirow{2}{*}{1,000} \\
\hline Retraída a esquerda & 18 & 19 & \\
\hline
\end{tabular}

NA: não se aplica; Teste McNemar.

Quanto à avaliação da FMR dos indivíduos avaliados foram observados valores estatisticamente significantes, sendo estes maiores no pós-treino quando comparado com o pré-treino para as variáveis PiMáx e PeMáx. A PiMáx apresentou aumento significante $(p=0,002)$ da mesma forma que a PeMáx $(p=0,006)$, como apresentado na Tabela 2 .
Por meio dos valores da espirometria nos dois momentos de avaliação, observamos também melhora quase significativa nas variáveis de capacidade vital funcional (CVF) e pico de fluxo expiratório (PFE) porém as outras variáveis espirométricas não obtiveram tal melhora (Tabela 2).

Foram encontradas correlações significativas entre PFE e PeMáx. A Figura 1 nos mostra uma correlação não significativa entre as variáveis PiMáx e PFE $(r=0,132$ e $p=0,578)$, porém, a figura 2 nos mostra uma correlação entre PFE e PeMáx estatisticamente significativa $(0,713$ e $p=0,0001)$.

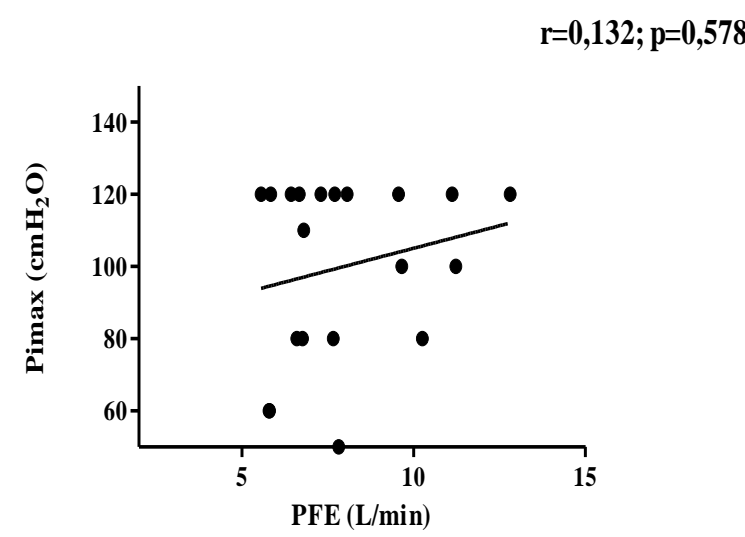

Figura 1. Relação entre PFE e PiMáx após a intervenção. Coeficiente de correlação de Spearman $p>0,05$.

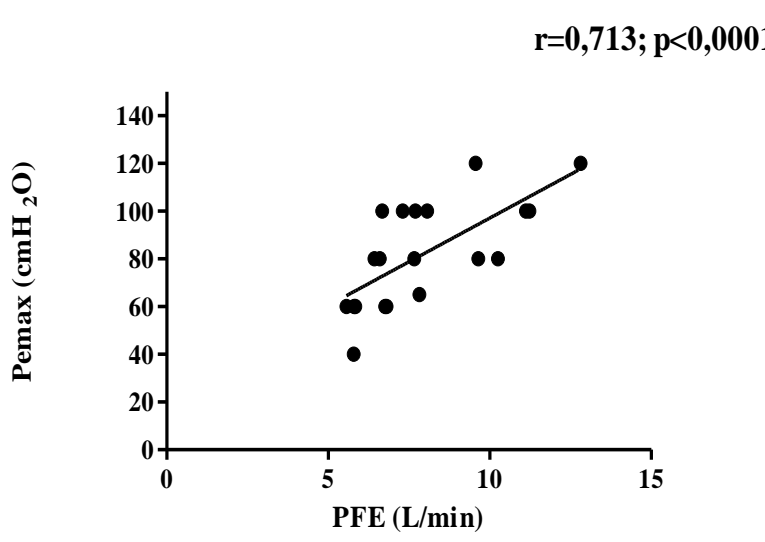

Figura 2. Relação entre PFE e PeMáx após a intervenção. Coeficiente de correlação de Spearman $p<0,05$. 
Tabela 2. Efeitos do programa de exercícios sobre a força muscular respiratória e função pulmonar

\begin{tabular}{|c|c|c|c|}
\hline & Pré-treino & Pós-treino & $p$ \\
\hline \multicolumn{4}{|l|}{ Força Muscular Respiratória } \\
\hline PiMáx $\left(\mathrm{cmH}_{2} \mathrm{O}\right)$, mediana (intervalo $\left.25-75 \%\right)$ & $80,0(72,5-120,0)$ & $115,0(80,0-120,0)$ & $0,002^{* b}$ \\
\hline PeMáx $\left(\mathrm{cmH}_{2} \mathrm{O}\right)$, media (DP) & $71,3(22,6)$ & $82,3(22,2)$ & $0,006^{* a}$ \\
\hline \multicolumn{4}{|l|}{ Índices espirométricos } \\
\hline $\operatorname{VEF}_{1}(\%$ pred), media (DP) & $100,7(15,1)$ & $98,2(14,6)$ & $0,074^{\mathrm{a}}$ \\
\hline CVF (\% pred), media (DP) & $101,0(18,0)$ & $98,8(17,5)$ & $0,055^{\mathrm{a}}$ \\
\hline PFE (L/min), mediana (intervalo $25-75 \%$ ) & $7,0(6,1-9,3)$ & $7,5(7,5-9,6)$ & $0,057^{b}$ \\
\hline $\mathrm{VEF}_{6}(\mathrm{~L} / \mathrm{min})$, media (DP) & $4,1(0,9)$ & $4,1(0,9)$ & $0,272^{\mathrm{a}}$ \\
\hline $\operatorname{VEF}_{1} / \mathrm{CVF}(\%)$, media (DP) & $90,6(6,0)$ & $90,3(6,8)$ & $0,661^{a}$ \\
\hline $\mathrm{FEF}_{25-75 \%}(\%$ pred$)$, media (DP) & $98,5(22,5)$ & $97,4(24,6)$ & $0,642^{\mathrm{a}}$ \\
\hline
\end{tabular}

Legenda: PiMáx: pressão inspiratória máxima; PeMáx: pressão expiratória máxima; $\mathrm{cmH}_{2} \mathrm{O}$ : centímetro de água; VEF1: volume expiratório forçado no primeiro segundo; CVF: capacidade vital forçada; PFE: pico de fluxo expiratório; VEF6: volume expiratório forçado nos primeiros seis segundos; VEF1/CVF: relação VEF1/CVF; FEF25-75\%: fluxo expiratório forçado entre 25 e 75\%; \%pred: porcentagem do predito; L/min: Litros por minuto; DP: desvio padrão; ${ }^{a}$ : Teste Wilcoxon; ${ }^{\mathrm{b}}$ : Teste t de Student para amostras pareadas; ${ }^{*}$ Diferença significativa $(p<0,01)$

\section{DISCUSSÃO}

A PiMáx e PeMáx apresentaram diferenças significativas quando comparados os períodos pré e pós treinamento. As variáveis espirométricas apresentaram-se dentro da normalidade e quando comparados os dois momentos houve melhora marginal à significância de PFE $(p=0,057)$ e CVF $(p=0,055)$. A postura dos indivíduos avaliados após 0 tratamento não evidenciou diferença significativa, porém foi observado uma importância clínica.

A PiMáx $(p=0,002)$ e PeMáx $(p=0,006)$ obtiveram aumento significativo após 0 tratamento com exercícios posturais. Resultados semelhantes foram descritos por Tomé et al. ${ }^{33}$, os quais diferiram apenas na frequência do treinamento, o qual foi realizado três vezes por semana, ou duas vezes semanais ${ }^{19}$. Evidencia-se, dessa forma, a melhora da FMR através do protocolo de exercícios utilizado em nosso estudo, porém, nossos achados demonstram que não é necessária frequência mínima de três ou duas vezes por semana, mas com apenas uma sessão semanal, composta de pelo menos um total de 10 sessões, é suficiente para promover tal melhora.

Quanto à postura desses jovens, nenhuma das variáveis mostrou melhora significativa após o treinamento, porém ao observar os valores descritivos da amostra notase uma melhora clínica destes, como por exemplo, no caso da escoliose em " $C$ " (Tabela 1). Um protocolo de exercícios embasados no método Pilates pode ser benéfico a pessoas com problemas posturais ${ }^{30}$, isso evidencia que pode haver melhora significativa de comprometimentos clínicos da escoliose, um maior número de sessões pode ser o diferencial nesses $\operatorname{casos}^{30}$.

A PFE e a CVF apresentaram respostas clínicas, com aumento dos valores após o tratamento. Mesmo sendo estes valores marginais à significância $(p=0,057$ e $p=0,055$ respectivamente), o estudo evidenciou uma melhora clínica, possivelmente devido á melhora da expansibilidade torácica ${ }^{34}$. Além disso, a correlação de PiMáx e PFE não mostrou correlação estatisticamente significante $(r=0,132$; $p=0,578$ ), porém a melhora da PeMáx apresentou uma correlação positiva com o aumento de PFE $(\mathrm{r}=0,703 ; p=0,0001)$, evidenciando a importância do treinamento sobre a musculatura expiratória para melhora de $\mathrm{PFE}^{35,36}$.

Todavia novos estudos são necessários dentro da temática, pois cada alteração postural pode interferir de uma forma diferente na mecânica ventilatória ${ }^{37}$, ou seja, estudos que apresentem de forma separada as alterações posturais, como a escoliose em " $C$ ", "S" e hipercifose ou hipocifose torácica, podem trazer uma melhor compreensão dessa mecânica e auxiliar no desenvolvimento de terapias mais eficazes.

Através do exposto, observa-se a importância dos exercícios posturais, baseados nos métodos de isostretching e Pilates, sobre a FMR, CVF e PFE, fato que mostra grande 
contribuição clínica, porém novos estudos devem ser realizados para observar os efeitos desses exercícios supracitados em outras populações, como por exemplo, indivíduos com DPOC, atletas, crianças ou ainda outras amostras populacionais.

Porém, algumas limitações podem ser citadas neste estudo. Este estudo foi um estudo de braço único, seria interessante usar como base as técnicas de exercício utilizadas, porém em grupos diferentes em um estudo de braço duplo e randomizado, sendo ideal para isso uma amostra maior.

Conclui-se que há melhora da FMR, realizando $o$ treinamento com exercícios posturais uma vez por semana associados a exercícios para mecânica ventilatória, da mesma forma que PFE e CVF também tiveram resultados positivos, porém em relação à postura não houve melhora considerável nos resultados.

\section{CONFLITO DE INTERESSE}

Os autores declaram não haver qualquer potencial conflito de interesse que possa interferir na imparcialidade deste trabalho científico.

\section{REFERÊNCIAS}

1. Santos NTO, Raimundo KC, Silva SA et al. Increased strength of the scapular stabilizer and lumbar muscles after twelve weeks of pilates training using the reformer machine: a pilot study. J Bodyw Mov Ther. 2017;21(1):74-80. DOI: https://doi.org/10.1016/i.jbmt.2016.05.005

2. Oliveira LC, Pires-Oliveira SA, Abucarub AC, Oliveira LS, Oliveira RG. Pilates increases isokinetic muscular strength of the elbow flexor and extensor muscles of older women: A randomized controlled clinical trial. J Bodyw Mov Ther. 2017;21(1):2-10. DOI: https://doi.org/10.1016/i.jbmt.2016.03.002

3. Sedrez JA, Rosa MIZ, Noll M, Medeiros FS, Candotti CT. Fatores de risco associados a alterações posturais estruturais da coluna vertebral em crianças e adolescentes. Rev Paul Pediatr. 2015;33(1):72-81. DOI: https://doi.org/10.1016/j.rpped.2014.11.012

4. Luz Jr M, Costa L, Fuhro F, Manzoni A, Oliveira $\mathrm{N}$, Cabral C. Effectiveness of mat pilates or equipment-based pilates exercises in patients with chronic nonspecific low back pain: a randomized controlled trial. Phys Ther. 2014;94(5):623-31. DOI: https://doi.org/10.2522/ptj.20130277

5. Wells C, Kolt G, Marshall P, Bialocerkowski A. Indications, Benefits, and risks of pilates exercise for people with chronic low back pain: a delphi survey of pilates-trained physical therapists. Phys Ther. 2014;94(6):806-17. DOI: https://doi.org/10.2522/ptj.20130568

6. Miyamoto G, Costa L, Galvanin T, Cabral C. Efficacy of the addition of modified pilates exercises to a minimal intervention in patients with chronic low back pain: a randomized controlled trial. Phys Ther. 2013;93(3):309-20. DOI: https://doi.org/10.2522/ptj.20120190

7. Araújo MEA, Silva EB, Vieira PC, Cader SA, Mello DB, Dantas EHM. Redução da dor crônica associada à escoliose não estrutural, em universitárias submetidas ao método Pilates. Motriz. 2010;16(4):958-66. DOI: https://doi.org/10.5016/1980$\underline{6574.2010 \mathrm{v} 16 \mathrm{n} 4 \mathrm{p} 958}$

8. Fernani DCGL, Cortez GM, Santos IA, et al. Anthropometric profile, physical activity level, degree of anxiety, and posture in college students. Fisioter Pesq. 2017;24(2):191-7. DOI: https://doi.org/10.1590/1809-

$\underline{\text { 2950/17160324022017 }}$

9. Kratenová J, Zejglicová K, Malý M, Filipová V. Prevalence and risk factors of poor posture in school children in the Czech Republic. J Sch Health. 2007;77(3):131-7. DOI: https://doi.org/10.1111/i.17461561.2007.00182.x

10. Straker L, O'Sullivan P, Smith A, Perry M. Computer use and habitual spinal posture in australian adolescents. Public Health Rep. 2007;122(5):634-43. DOI: https://doi.org/10.1177/003335490712200511

11. Badaró A, Nichele L, Turra P. Investigação da postura corporal de escolares em estudos brasileiros. Fisioter Pesq. 2015;22(2):197-204.

12. Salve M, Bankoff A. Postura corporal: um problema que aflige os trabalhadores. Rev Bras Saúde Ocup. 2013;28(105):91-103. DOI: 
https://doi.org/10.1590/S0303-

\section{$\underline{76572003000100010}$}

13. Moreno M, Catai A, Teodori R, Borges B, Cesar $M$, Silva $E$. Effect of a muscle stretching program using the Global Postural Re-education method on respiratory muscle strength and thoracoabdominal mobility of sedentary young males. J Bras Pneumol. 2007;33(6):679-86. DOI: https://doi.org/10.1590/S1806-

$\underline{37132007000600011}$

14. American Thoracic Society / European Respiratory Society ATS / ERS Statement on Respiratory Muscle Testing. Am J Respir Crit Care Med. 2002;166:518-624. DOI: https://doi.org/10.1164/rccm.166.4.518

15. Castro CAP. Sociedade Brasileira de Pneumologia e Tisiologia (SBPT). I Consenso Brasileiro sobre Espirometria. J Bras Pneumol. 1996;22:64-105.

16. Carvalho A, Assini T. Aprimoramento da capacidade funcional de idosos submetidos a uma intervenção por isostretching. Braz J Phys Ther. 2008;12(4):268-73. DOI: https://doi.org/10.1590/S1413-

$\underline{35552008000400004}$

17. Rezende M, Carvalho L, Feliphe R, Rocha C, Silva $V$, lunes $D$. Efeitos do método isostretching na função respiratória e postura de respiradores bucal. Cons Saude. 2016;15(1):89-95. DOI: https://doi.org/10.5585/conssaude.v15n1.5959

18. Giacomini $M$, da Silva A, Weber L, Monteiro $M$. The pilates method increases respiratory muscle strength and performance as well as abdominal muscle thickness. J Bodyw Mov Ther. 2016;20(2):258-64. DOI: https://doi.org/10.1016/j.jbmt.2015.11.003

19. Lopes E, Ruas G, Patrizz L. Efeitos de exercícios do método Pilates na força muscular respiratória de idosas: um ensaio clínico. Rev bras geriatr gerontol. 2014;17(3):517-23. DOI: https://doi.org/10.1590/1809-9823.2014.13093

20. Silva ACLG, Mannrich G. Pilates na reabilitação: uma revisão sistemática. Fisioter Mov. 2017;22(3):144-55. https://periodicos.pucpr.br/index.php/fisio/articl e/view/19479. Accessed June 6, 2019.
21. Sanglard RCF. A influência do isostretching nas alterações dos parâmetros da marcha em idosos. Fisioter Bras. 2018;6(4):255-60. DOI: https://doi.org/10.33233/fb.v6i4.2001

22. SISVAN - Sistema de Vigilância Alimentar e Nutricional. Curvas de Crescimento da Organização Mundial da Saúde-OMS, 2017. Acesso em: 18 maio 2019. Disponível em: http://nutricao.saude.gov.br/sisvan.Php?Conteud $\mathrm{o}=$ curvas_cresc_oms

23. Benedetti TRB, Antunes PDC, Rodriguez-añez CR, Mazo GZ, Petroski ÉL. Reprodutibilidade e validade do Questionário Internacional de Atividade Física ( IPAQ ) em homens idosos. Rev Bras Med Esporte. 2007;13(6):11-6. DOI: https://doi.org/10.1590/S1517$\underline{86922007000100004}$

24. Liposcki D, Rosa Neto F, Savall A. Validação do conteúdo do instrumento de avaliação posturalIAP. Rev Digital. 2007;12(109):1-7.

25. Neder JA, Andreoni S, Lerario MC, Nery LE. Reference values for lung function tests . II . Maximal respiratory pressures and voluntary ventilation. Braz J Med Biol Res. 1999;32:719-27. DOI: $\quad$ https://doi.org/10.1590/S0100$\underline{\text { 879X1999000600007 }}$

26. Costa D, Gonçalves HA, Lima LP De, Ike D, Cancelliero KM, Montebelo MI de L. Novos valores de referência para pressões respiratórias máximas na população brasileira. J Bras Pneumol. 2010;36(3):306-12. DOI: https://doi.org/10.1590/S1806$\underline{37132010000300007}$

27. Miller MR, Hankinson J, Brusasco V, et al. Standardisation of spirometry. Eur Respir J. 2005;26(2):319-38. DOI: https://doi.org/10.1183/09031936.05.00034805

28. Cepeda C, Rodacki A, Persch L, Silva P, Bulba $S$, Dressler V. Efeitos do método isostretching sobre parâmetros morfológicos e sobre um conjunto de testes motores em idosas. Rev Bras Cineantropom Desempenho Hum. 2013;15(5):604-15. DOI: https://doi.org/10.5007/1980$\underline{0037.2013 v 15 n 5 p 604}$ 
29. Monte-Raso V, Ferreira $P$, de Carvalho $M$, Rodrigues J, Martins C, lunes D. Efeito da técnica isostretching no equilíbrio postural. Fisioter Pesq. 2009;16(2):137-42.

DOI: https://doi.org/10.1590/S1809-

$\underline{29502009000200008}$

30. Cruz JC, Liberali R, Cruz TMF, Netto MIA. The Pilates method in the rehabilitation of musculoskeletal disorders : a systematic review. Fisioter Em Mov. 2016;29(3):609-22. DOI: https://doi.org/10.1590/1980-

$\underline{\text { 5918.029.003.AO19 }}$

31. Macedo C, Debiagi P, Andrade F. Efeito do isostretching na resistência muscular de abdominais, glúteo máximo e extensores de tronco, incapacidade e dor em pacientes com lombalgia. Fisioter Mov. 2010;23(1):113-20. DOI: https://doi.org/10.1590/S0103-

$\underline{51502010000100011}$

32. Sinzato C, Taciro C, Pio C, de Toledo A, Cardoso J, Carregaro R. Effects of 20 sessions of Pilates method on postural alignment and flexibility of young women: pilot study. Fisioter Pesq. 2013;20(2):143-50. DOI: https://doi.org/10.1590/S1809-

29502013000200008

33. Tomé F, Ferreira CB, Cornelli RJB, Carvalho AR de. Lombalgia crônica: comparação entre duas intervenções na força inspiratória e capacidade funcional. Fisioter Mov. 2012;25(2):263-72. DOI: https://doi.org/10.1590/S0103-

$\underline{51502012000200003}$

34. Freire E, Ribeiro A, Zago L. Fortalecimento muscular e expansibilidade pulmonar após o uso da técnica de isostretching. Inter Faces Cient. 2016;4(3):31-8.

DOI:

https://doi.org/10.17564/2316-

$\underline{3798.2016 v 4 n 3 p 31-38}$

35. Enright S, Unnithan V, Heward C, Withnall L, Davies D. Effect of high-intensity inspiratory muscle training on lung volumes, diaphragm thickness, and exercise capacity in subjects who are healthy. Phys Ther. 86(3):345-54. DOI: https://doi.org/10.1093/pti/86.3.345

36. Caruso $P$, de Albuquerque A, Santana $P$, et al. Diagnostic methods to assess inspiratory and expiratory muscle strength. I Bras Pneumol. 2015;41(2):110-23.

DOI: https://doi.org/10.1590/S1806-

$\underline{37132015000004474}$

37. Contesini A, Garcia Jr A, Caromano F. Influência das variações da postura sentada na função respiratória: revisão de literatura. Fisioter Mov. 2011;24(4):757-67.

DOI:

\section{1}

